\section{$\underset{\substack{\text { hommes } \\ \text { \& migrations }}}{ }$}

\section{Hommes \& migrations}

Revue française de référence sur les dynamiques

migratoires

1277 | 2009

France - Allemagne

\title{
La lutte contre le racisme des "minorisés" en France et en Allemagne depuis les années 1980
}

\section{Soline Laplanche-Servigne}

\section{(apenEdition \\ Journals}

\section{Édition électronique}

URL : http://journals.openedition.org/hommesmigrations/162

DOI : 10.4000/hommesmigrations. 162

ISSN : 2262-3353

\section{Éditeur}

Musée national de l'histoire de l'immigration

\section{Édition imprimée}

Date de publication : 1 janvier 2009

Pagination : $56-66$

ISSN : 1142-852X

\section{Référence électronique}

Soline Laplanche-Servigne, "La lutte contre le racisme des "minorisés" en France et en Allemagne depuis les années 1980 », Hommes \& migrations [En ligne], 1277| 2009, mis en ligne le 29 mai 2013, consulté le 19 avril 2019. URL : http://journals.openedition.org/hommesmigrations/162 ; DOI : 10.4000/hommesmigrations.162 


\section{La lutte contre le racisme des "minorisés" en France et en Allemagne depuis les années 1980}

Par Soline Laplanche-Servigne, doctorante, Institut d'études politiques de Paris

Dans la dernière décennie du $X X^{e}$ siècle, les victimes du racisme, dotés de leurs propres organisations, ont pris la parole dans l'espace public et se sont autodésignés des Noirs et des Indigènes en France,

ou des Kanaks en Allemagne. En mobilisant l'histoire et leur vécu, ils ont ouvert une bataille sémantique et posé leur inégalité de statut dans les deux sociétés. Le débat s'est répandu au sein du mouvement antiraciste, le discours officiel a été contesté.

Aujourd'hui, une redéfinition du racisme est engagée et les représentations de l'autochtone face à l'allochtone évoluent vers la reconnaissance de sociétés davantage multiculturelles. 
La proposition d'amendement, dit Mariani ${ }^{(1)}$, autorisant le recours aux tests ADN lors de la délivrance des visas de plus de trois mois dans le cadre d'un regroupement familial a ravivé en France les questionnements sur l'attitude adoptée par un État face à l'Autre immigré, au non-national. Elle a soulevé l'indignation de nombreuses organisations antiracistes. Ce débat souligne à nouveau à quel point les préoccupations de l'antiracisme sont aujourd'hui tributaires d'un enchevêtrement du national et du supranational, de la situation interne aux États-nations et des facteurs externes que sont les mouvements migratoires ou les conflits internationaux.

Il résulte de cet enchevêtrement une forme de malaise en France et en Allemagne (et plus généralement en Europe) à l'idée de lier la question de l'immigration, des discriminations et celle du racisme. On observe, d'une part, une volonté de plus en plus affichée par les gouvernements, au moins dans les discours, de lutter contre les discriminations, et d'autre part, des restrictions accrues vis-à-vis de l'immigration, et une difficulté croissante à parler de racisme et d'antiracisme. Les gouvernements français et allemand évoquent plus volontiers la "promotion de la diversité", que la "lutte contre le racisme", et les associations

L'antiracisme est un thème qui fait beaucoup plus largement partie du débat public en France qu'en Allemagne, où il reste surtout cantonné à la scène politique de gauche. elles-mêmes refusent parfois de se qualifier d'antiracistes, ou se voient dénier ce qualificatif par leurs détracteurs estimant parfois qu'elles ne font pas de l'antiracisme ${ }^{(2)}$. Cette difficulté croissante à parler d'antiracisme et à le définir est notamment liée à la complexification du paysage du racisme. Comme le souligne Michel Wieviorka, le phénomène du racisme se complexifie à la fois à l'intérieur des sociétés occidentales, "en fonction de leur évolution interne, et notamment de leur fragmentation culturelle et sociale", et à la fois en fonction de logiques supranationales, qui résultent d'événements externes à ces sociétés (le conflit israélo-palestinien, les attentats du 11 septembre, etc. $)^{(3)}$.

Il s'agit ici de rendre compte de la parole de "minorisés" en France et en Allemagne sur la question du racisme et des discriminations raciales, en se gardant d'une définition préconçue de ce que serait l'antiracisme aujourd'hui. Car en réalité, analyser le point de vue de "minorisés" luttant en France et en Allemagne contre les discriminations raciales ne revient pas à analyser seulement une lutte "contre". En effet, les "minorisés" qui cherchent à faire entendre leur voix - sans forcément de succès - dans l'espace public et politique, ne cherchent pas seulement à dénoncer 
les discriminations raciales dont ils sont potentiellement les victimes, ils expriment également une quête de reconnaissance, contribuant à diffuser au sein de leur société les éléments d'une définition renouvelée du "nous" national.

\section{La parole des minorisés entre antiracisme et lutte mémorielle contre les discriminations raciales}

Il faut tout d'abord remarquer que l'antiracisme est un thème qui fait beaucoup plus largement partie du débat public en France qu'en Allemagne, où il reste surtout cantonné à la scène politique de gauche. En France, on peut se souvenir de la très forte médiatisation de l'association SOS-Racisme dans les années 1980. On peut aussi citer les dissensions au sein de l'antiracisme associatif lors de la tentative d'organisation d'une manifestation antiraciste unitaire en novembre $2004^{(4)}$; ou encore, les polémiques de 2006 autour des prises de position du président du MRAP $^{(5)}$ contre l' "islamophobie”((). Enfin, depuis 2005, la légitimité d'organisations militantes de lutte contre le racisme ou contre les discriminations raciales à se désigner selon des terminologies liées à une couleur de peau, ou rappelant un lien à l'immigration par l'emploi des termes "Noirs" ou "Indigènes", est débattue.

On a ainsi vu émerger dans l'espace public de façon très médiatisée le Conseil représentatif des associations noires (Cran) ${ }^{(7)}$ en novembre 2005, et les Indigènes de la République, avec la publication de 1'“Appel des indigènes de la République" ${ }^{\text {"(8), lancé }}$ sous forme de pétition sur l'Internet en janvier 2005. Si la Marche pour l'égalité et contre le racisme, en 1983, avait déjà fait apparaittre sur la scène publique les jeunes issus de l'immigration (principalement maghrébine) qui furent appelés les "beurs", leurs revendications ne faisaient pas appel, au contraire des nouvelles associations, à l'histoire et à la mémoire comme ressources mobilisatrices.

En Allemagne, des organisations de minorisés luttant contre les discriminations raciales existent également, mais elles sont beaucoup moins présentes dans l'espace public. L'organisation Initiative Schwarze Menschen in Deutschland (Initiative des Noirs d'Allemagne) - créée en 1986 sous le nom initial d'Initiative Schwarze Deutsche (Initiative des Allemands noirs) ${ }^{(9)}$ est dans son discours assez similaire au Cran. On peut aussi mentionner Kanak Attak ${ }^{(10)}$ qui rejoint la ligne militante des Indigènes de la République en France - les termes de "Kanak" et d'“Indigène" évoquant une même vision des inégalités de statut dans les deux sociétés. Plus récemment, la voix de minorisés, protestant contre le racisme, s'est faite entendre avec la campagne menée par l'Afrika Rat, ou Conseil africain, peu avant le début de la Coupe du monde de football en mai 2006. Cette fédération de vingt-cinq asso- 
ciations avaient alors appelé à "enfin prendre au sérieux" les violences racistes contre les gens d'origine africaine, et avait fait état de l'existence dans la région de BerlinBrandenburg de "No-go-areas", notamment à l'Est, où elle déconseillait aux gens de couleur de se rendre en raison de risques d'agressions racistes. La création de l'Afrika Rat et la campagne sur les "No-go-areas" suivaient l'agression à Potsdam, petite ville à l'est de Berlin, d'un Allemand d'origine éthiopienne ${ }^{(11)}$.

\section{Racisme et discriminations : le point de vue des victimes}

Ce qui fait débat, dans les deux pays, c'est toujours la question de la qualification des actes : qu'est-ce qui relève du racisme? Telle agression a-t-elle des causes racistes ou antisémites ? Les groupes de minorisés, en lutte contre le racisme et les discriminations raciales que nous avons cités, regrettent généralement la minimisation du phénomène du racisme par les politiques, et l'absence de reconnaissance d'un racisme structurel, ancré dans la société, et non pas seulement exprimé à la marge par des extrémistes. L'Afrika Rat, l'Initiative Schwarze Deutsche ou les Indigènes de la République déplorent ainsi que les pouvoirs publics mènent une lutte principalement contre l'extrémisme de droite, ou ne reconnaissent pas le racisme institutionnel, les préjugés et stéréotypes répandus dans toute la société. Ce qui distingue ce type d'organisations de lutte contre le racisme et contre les discriminations raciales, par rapport aux organisations antiracistes blanches (telles qu'en France SOS-Racisme, la LDH, etc.), c'est la place accordée à l'histoire et à la mémoire comme ressource mobilisée dans la lutte contre les discriminations raciales. Ces organisations, bien que dans des registres différents, font toutes appel à l'histoire pour expliquer les discriminations et les préjugés dont leurs membres se sentent victimes. Qu'il s'agisse de la mémoire de la colonisation, de l'esclavage ou de l'immigration de travail subis par leurs parents ou par leurs ancêtres.

Mais à partir de cette caractéristique commune, qui donne sa particularité à ce type de mobilisations de minorisés, certaines associations privilégient un discours d'analyse des causes du racisme et de ses racines, un discours de dénonciation des représentations mentales à l'égard des descendants issus de l'immigration - c'est le cas des Indigènes de la République ou de Kanak Attak. D'autres, de façon peutêtre plus pragmatique, privilégient le combat sur la base des conséquences, en l'occurrence les discriminations observables dans la société à l'égard des étrangers ou des nationaux de couleur. C'est ce qui conduit les membres d'une organisation 
telle que le Cran à se défendre de faire de l'antiracisme et à définir leur action comme une action de lutte non pas contre le racisme, mais contre les discriminations ethno-raciales. Patrick Lozès, son président, explique ainsi la distinction opérée par le Cran entre racisme et discrimination - son action étant déterminée par le second concept :

"Les discriminations c'est le racisme des racistes, plus le racisme des non-racistes, et [...] quand vous parlez de racisme, les gens disent "mais je suis pas raciste", point à la ligne. "Je ne suis pas raciste, mais je ne vous loue pas mon appartement" [...] Parce que le racisme, c'est un concept [...], c'est un sentiment [...], mais tant que vous n'avez pas de passage à l'acte, c'est un sentiment! alors que les discriminations, voilà quelque chose de concret! Et le point cardinal, c'est que ça peut être objectivé." ${ }^{(12)}$

Que ces mobilisations insistent sur une lutte antiraciste ou sur une lutte antidiscriminations, elles contribuent en tout cas à diffuser dans l'espace public les éléments d'une redéfinition du "nous" national et de nouvelles modalités d'appartenance nationale.

\section{Le rôle des organisations de minorisés dans la redéfinition de l'identité nationale}

Si la France et l'Allemagne sont depuis plusieurs décennies devenues des sociétés multiculturelles, la prise de conscience ou l'acceptation de cette diversité ethnique de la population a pris du temps et s'est mêlée aux questionnements sur l'identité nationale. Un phénomène particulièrement aigu en Allemagne après la chute du mur de Berlin et la réunification des deux Allemagnes. Celui-ci s'est accompagné d'un double mouvement, d'un côté un nouvel élan national, s'accompagnant parfois de violences xénophobes perpétrées contre des demandeurs d'asile, à Solingen, à Rostock ou à Mölln ; d'un autre côté des réactions antinationalistes, comme le mouvement des "Antideutsche" (Anti-Allemands) et la campagne "Nie wieder Deutschland"(Plus jamais l'Allemagne). En France, les succès électoraux du Front national, à partir du milieu des années 1980, dénotaient la réceptivité d'une partie de la population à des thèses nationalistes et xénophobes, et plus récemment, c'est sous la forme de discussions autour du passé colonial de la France qu'a émergé à nouveau la question du lien entre immigration (postcoloniale) et identité nationale.

Quel est le rôle des minorisés comme acteurs de ces questionnements autour de l'identité nationale et de la lutte contre les formes de repli nationaliste et xénophobe? En France et en Allemagne, le mouvement antiraciste a connu un 
essor nouveau à partir des années 1980, en réaction au durcissement de la loi sur les étrangers en Allemagne, et en France face aux succès électoraux du Front national notamment, mais aussi face aux violences policières contre les jeunes issus de l'immigration. Toutefois, au sein du mouvement antiraciste, les minorisés ont d'abord été principalement objet, cantonnés à leur statut de victime, plutôt que sujet de la lutte antiraciste. En Allemagne, il a existé peu d'organisations (ou de tentatives d'organisations) regroupant à la fois des Allemands et des migrants ou descendants de migrants. En France, la Marche pour

En Allemagne peu d'organisations (ou de tentatives d'organisations) regroupant à la fois des Allemands et des migrants ou descendants de migrants. l'égalité et contre le racisme de 1983 a fait apparaître dans l'espace public la deuxième génération issue de parents immigrés (maghrébins principalement), qu'on a nommé les beurs. Mais par la suite, même si certains ont fait partie de l'association SOS-Racisme, on n'assiste pas à l'émergence d'un mouvement antiraciste des minorisés.

En France, c'est très récemment qu'ont émergé dans l'espace public des organisations revendiquant une lutte contre le racisme ou contre les discriminations ethno-raciales qui ne fassent pas abstraction du rôle joué par la couleur de la peau ou du vécu du racisme et des discriminations par les immigrés. Si ce type de mobilisations provoque des polémiques, elles profitent aujourd'hui en France, de structures d'opportunité qui n'existent pas en Allemagne. En effet, alors qu'on entend en France depuis quelques années un discours, de la part des politiques, sur la nécessité de promouvoir l'accès des “minorités visibles”(des Français non blancs) à des lieux de pouvoir, on évoque encore en Allemagne les "étrangers" (Ausländer) - et tout de même peu à peu les "jeunes issus de l'immigration" (mit Migrationshintergrund). Pour le moment, la prise en compte des minorisés se traduit plutôt en termes de promotion de la participation politique des migrants, à travers leur représentation dans des instances locales. On peut citer ainsi, la création en 2003 par le sénat de Berlin d'un conseil régional pour les questions d'intégration et de migration (Landesbeirat für Integrations- und Migrationsfragen), dont font partie six représentants des migrants. On peut aussi noter la déclaration de Maria Böhmer, déléguée du gouvernement fédéral à la migration, aux réfugiés et à l'intégration, à l'occasion du sommet de l'intégration de juillet 2007 : "nous ne parlons plus des migrants, nous parlons avec eux". Quel rôle les organisations de minorisés luttant contre les discriminations raciales jouent-elles dans la façon de désigner cet Autre avec lequel il faut dialoguer ou que l'on veut rendre visible ? 


\section{L'autodésignation des minorisés et les nouvelles représentations de l'autochtone}

Le sociologue Didier Fassin souligne la difficulté que l'on a en France - mais cette remarque pourrait s'appliquer à l'Allemagne également - à nommer les individus victimes de discriminations raciales: "Les populations, comme l'on dit, celles et ceux dont on parle, qui sont victimes de discriminations, qui font l'objet parfois de politiques spécifiques,

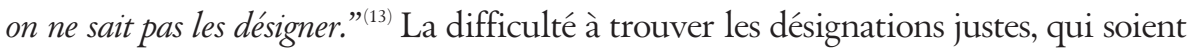
acceptables et acceptées par tous, en premier lieu par les individus touchés par les discriminations, est en effet flagrante aujourd'hui. C'est là un des rôles que peuvent jouer les organisations militantes de minorisés : par les désignations qu'elles se choisissent, elles dépeignent leur vision de la réalité sociale et introduisent, dans l'espace public, de nouvelles façons de nommer les victimes de discriminations raciales. Le Cran en France a largement contribué à diffuser dans l'espace public l'utilisation de la dénomination "Noirs" pour parler des Français noirs. En Allemagne, ISD évoque les "Afro-deutsche". Kanak Attak, sur le mode de la provocation, distingue les "Kanak" des "Bio-Deutsche"(Allemands de souche).

Ces organisations contribuent à rendre moins euphémique le discours sur les minorisés. Le porte-parole du Cran, Louis-Georges Tin, décrit ainsi son arrivée à une des premières réunions où fut évoquée la création du futur Cran - dont les membres fondateurs étaient rassemblés au départ au sein du Cercle d'action pour la promotion de la diversité en France (Capdiv) : "Quand je suis arrivé le premier jour, jai dit : mais en fait vous n'êtes pas divers, vous êtes Noir!”(14).

On peut aussi donner l'exemple des militants du Mouvement des indigènes de la République (MIR), qui revendiquent le caractère politique de leur autodésignation comme "Indigènes", au-delà de l'hétérogénéité des origines des individus composant ce mouvement antiraciste. La porte-parole du MIR, Houria Bouteldja, explique ainsi la dénomination d'Indigène : "Quel est le nom qu'on peut se donner? On a tout : les Arabes, les musulmans, les Africains, les Noirs, les immigrés, les enfants d'immigrés, les gens issus de l'immigration, les je-ne-sais-pas-quoi... [...] le terme Indigène est venu parce qu'au bout d'un moment il faut pouvoir nommer la réalité. Et la réalité, c'est qu'on ne sera jamais français, puisquêetre français, c'est appartenir à une espèce de caste. C'est une espèce d'honneur et n'est pas français qui veut. [...] Nous, on ne peut pas y prétendre parce qu'on est trop basanés et on ne sera jamais assez blancs pour être français, quoiqu'on dise et quoiqu'on fasse. [...] Et cette réalité, il faut la nommer, on ne peut pas être français donc on est des Indigènes! "(15)

Si la tonalité et la teneur du combat mené par le MIR sont assez différentes de celles du Cran, les militants des deux organisations ont en commun de chercher, 
à travers l'emploi d'une terminologie, à nommer un groupe d'individus dont la caractéristique commune est la discrimination (ou potentielle discrimination) vécue au sein de la société. C'est la situation d'inégalité vécue qui crée cette catégorie d'individus. Les militants du MIR indiquent par l'emploi du terme d'Indigène pour s'autodésigner qu'ils ne s'estiment pas perçus comme des Français à part entière, en raison de leur lien à une immigration postcoloniale. Les membres du Cran, par la revendication de l'utilisation du terme Noirs pour se désigner, soulignent que c'est en raison de leur couleur de peau, qu'ils s'estiment discriminés au sein de la société française, majoritairement blanche.

Parler de Noirs en France,
plutôt que de citoyens
français uniquement,
introduit une
reconnaissance nouvelle
de la couleur de peau
comme facteur de
discrimination potentielle
des individus.

L'apparition d'autres dénominations peut être signalée, comme en Allemagne l'expression anglophone de "People of Colour", utilisée par exemple par le Réseau antidiscrimination de Berlin (Antidiskriminierungsnetzwerk Berlin). Si cette organisation n'est pas exactement une organisation de minorisés, elle est fédérée par la Confédération turque de Berlin-Brandenburg (Türkischer Bund in BerlinBrandenburg), et regroupe diverses associations de migrants. L'expression (orthographiée cette fois "People of Color") est également utilisée par des chercheurs et militants associatifs souhaitant analyser dans un ouvrage collectif le racisme, la politique culturelle et les modes de résistance au racisme en Allemagne, selon la perspective d'individus eux-mêmes concernés par le problème $^{(16)}$. Les auteurs revendiquent cette désignation comme un concept politique de solidarité, comme une "plate-forme commune" permettant de s'adresser "à tous les membres de communautés racisées et opprimées"

L'apparition d'associations ou d'ouvrages revendiquant, pour décrire la réalité sociale, l'utilisation de dénominations liées à une couleur de peau, à un phénotype, indique sans doute une évolution progressive de la façon de considérer les situations d'inégalités dans les sociétés française et allemande, entre la population blanche et la population de couleur. Si l'on ne peut prévoir la capacité de diffusion à long terme par ces associations ou par ces chercheurs de telles dénominations dans l'espace public, on peut toutefois envisager le rôle que ces acteurs pourraient jouer, et jouent parfois déjà, sur les représentations cognitives des individus. Une membre du Cran explique par exemple : "le mot Noirs, jai eu du mal un petit peu à l'intégrer hein. Parce que... parce qu'on n'en parlait pas et... finalement, euh... enfin moi, jai tendance à dire Black, peut-être parce que c'est de ma 
génération, et finalement c'est très très bien d'essayer de banaliser ce terme [de Noirs]." ${ }^{(18)}$ Comme le remarque Riva Kastoryano, la question qui se pose dans des sociétés devenues multiculturelles, est de savoir "Quelle identité devient légitime pour une reconnaissance publique : comment se définit la légitimité de la différence et de l'altérité, en fonction de quels critères et par quels mécanismes ? "(19) La façon de nommer les différentes composantes d'une population est partie prenante de la reconnaissance que l'on choisit d'accorder aux différents groupes de population et aux causes de leur vulnérabilité face à la discrimination raciale. Parler de Noirs en France, plutôt que de citoyens français uniquement, introduit une reconnaissance nouvelle de la couleur de peau comme facteur de discrimination potentielle des individus.

Les dénominations, autodésignations, choisies par ces organisations de lutte contre les discriminations raciales, peuvent contribuer à faire évoluer les représentations que l'on a, dans ces sociétés, de l'autochtone et de l'allochtone. Peu à peu, le Français (ou l'Allemand) ne sera peut-être plus imaginé comme forcément blanc.

\section{Redéfinitions du racisme et de l'antiracisme, la prise en compte des victimes}

En conclusion, nous aimerions nous interroger sur l'évolution de la définition du racisme et de l'antiracisme dans nos sociétés européennes. Gérard Noiriel souligne une "redéfinition des termes "antisémitisme" et "racisme" qui résulte du triomphe des sondages d'opinion dans ce domaine également. [...] Ces mots sont nés au départ pour désigner des discours et des programmes politiques, assumés par des acteurs qui étaient maîtres de leurs propos publics. [...] Depuis 1990, le "racisme" et l"antisémitisme" sont devenus des "opinions personnelles" recueillies par des instituts de sondage [...]" ${ }^{(20)}$ Notre recherche sur les mobilisations antiracistes et antidiscriminations de minorisés nous incite aux réactions suivantes : les définitions du racisme et de l'antisémitisme ont en effet évolué de définitions strictement circonscrites à des programmes politiques, à une prise en compte des représentations des individus sur la question (on interroge alors par exemple les gens sur leurs représentations par rapport à tel ou tel groupe de population). Mais cette évolution doit être reliée à la progressive prise en considération, non plus uniquement des auteurs du racisme et l'antisémitisme, mais aussi des victimes. Cette prise en considération des victimes signifie notamment une attention accordée à leur ressenti et à leurs perceptions. Or on ne peut faire abstraction de la perception, de la part des populations minorisées, d'un racisme ordinaire, qui relève effectivement d'opinions et de 
stéréotypes. Bien sûr, élargir la définition du racisme, par la prise en compte des représentations des individus - que ce soient celles des victimes ou celles des auteurs (conscients ou non) - complexifie la circonscription du phénomène. Le racisme ne renvoie plus seulement à une idéologie, il est aussi défini à partir de ses conséquences. Des pratiques peuvent donc être considérées comme racistes, sans que la personne qui en est l'auteur en ait eu l'intention ou en ait eu conscience. Les associations de lutte contre le racisme, particulièrement lorsqu'elles sont dirigées par des individus minorisés, sont parfois accusées d'exagérer l'ampleur du racisme et des discriminations, ou de banaliser les termes de racisme et d'antisémitisme. Toutefois, il nous semble qu'on ne peut (doit) occulter le fait qu'aujourd'hui, être Européen reste largement associé au fait d'être Blanc et chrétien. Dès lors, s'il peut être critiquable de galvauder les termes de racisme et d'antiracisme, en y incorporant sans discernement les subjectivités de chacun, il convient cependant de ne pas oublier les privilèges du "sac à dos invisible" décrits par Peggy McIntosh ${ }^{(21)}$, et dont jouissent inconsciemment les "non racialisés" en Europe. Ces systèmes invisibles de privilèges qui avantagent les Blancs nécessitent que l'on reste attentif au vécu des minorisés et qu'un dialogue se perpétue afin que chacun se sente un "partenaire à part entière"(22) au sein de la société dans laquelle il choisit de vivre. 


\section{Notes}

1. L'amendement a été adopté par la commission des lois de l'Assemblée nationale le 12 septembre 2007. Le projet de loi de Brice Hortefeux sur l'immigration, contenant cet amendement, a été adopté le 20 septembre 2007 par l'Assemblée nationale, et l'amendement controversé, autorisant le recours aux tests ADN, a été sous-amendé et voté par les sénateurs le 3 octobre 2007.

2. Cette critique a notamment été adressée au Mouvement des indigènes de la République. Ses détracteurs ont accusé les militants du MIR de produire eux-mêmes des analyses et des discours racistes, dans leur façon de dénoncer un racisme structurel en France.

3. Voir Wieviorka, Michel, "Racisme : changements dans le phénomène, changements dans l'analyse",

Working paper, n40, présenté à l'Université américaine de Paris le 5 décembre 2006.

4. Manifestation organisée à l'appel de la LDH (Ligue des droits de l'homme), du Mrap et des principales organisations syndicales, mais qui a rencontré l'opposition du Crif, de la Licra, de SOS-Racisme et de Ni Putes ni Soumises, qui ont souligné la nécessité d'un combat spécifique contre l'antisémitisme, et ont refusé la présence de l'UOIF

(Union des organisations islamiques de France) dans le cortège de la manifestation.

5. Mouvement contre le racisme et pour l'amitié entre les peuples (depuis 1977); à ses origines, en 1949 son intitulé était : Mouvement contre le racisme, l'antisémitisme et pour la paix.

6. Le Mrap a essuyé des critiques - notamment par une partie de ses militants - pour avoir utilisé le terme d' "islamophobie" et il a été reproché au président du mouvement, Mouloud Aounit, de privilégier par ses prises de position la lutte contre le racisme antimusulman, au détriment d'autres racismes, notamment l'antisémitisme. Mouloud Aounit s'en est défendu en indiquant que c'était "bien au nom de [son] attachement indéfectible à l'universalisme qu' [il] souhaite que l'islamophobie soit inscrite sur l'agenda antiraciste", il a aussi affirmé son attachement à un antiracisme "indivisible".

7. Le Cran a été créé en France en novembre 2005, en tant que fédération d'une soixantaine d'associations noires de France et a pour objectif de lutter contre les discriminations "ethno-raciales" et "pour le devoir de mémoire".

8. "Nous sommes les Indigènes de la République!", cet Appel dénonçait "l'oppression et les discriminations produites par la République postcoloniale" et appelait à la tenue d' "assises de l'anticolonialisme", et à une "première Marche des Indigènes de la République"

le 8 mai 2005, pour poursuivre "le combat anticolonial".

9. Le changement de nom s'est effectué en 1995.

10. Cette association existe depuis 1998 et prône "une nouvelle attitude des migrants de toutes les générations",

"autonome [...] et sans conformisme". Voir : www.kanak-attak.de/ka/about.html.

11. Il s'en est suivi en Allemagne une polémique sur la nature raciste ou non de cette agression.

12. Extrait d'un entretien réalisé en septembre 2007.

13. Fassin, Didier, "Nommer, interpréter. Le sens commun de la question raciale", in Fassin, Didier, et Fassin, Éric,

De la question sociale à la question raciale? Représenter la société française, La Découverte, Paris, 2006, p.24.

14. Extrait d'un entretien réalisé en septembre 2007.

15. Bouteldja, Houria, "Féminisme et antiracisme", Contre Temps, "Postcolonialisme et immigration", n`16, mai 2006, p. 89.

16. Kien, Nghi Ha, Nicola, Lauré al-Samarai, Sheila Mysorekar (Hg.), re/visionen. Postkoloniale Perspektiven von People of Color auf Rassismus, Kulturpolitik und Widerstand in Deutschland, Unrast, Münster, 2007.

17. Kien, Nghi Ha, Nicola, Lauré al-Samarai, Sheila, Mysorekar, (Hg.), ibid., p. 35.

18. Extrait d'un entretien réalisé en décembre 2006.

19. Kastoryano, Riva (dir.), Les Codes de la différence, Presses de Sciences Po, 2005, p.15.

20. Noiriel, Gérard, Immigration, antisémitisme et racisme en France $\left(X I X^{e}-X X^{e}\right.$ siècle). Discours publics, humiliations privées, Fayard, Paris, 2007, p.654.

21. McIntosh, Peggy, "White Privilege : Unpacking the Invisible Knapsack", extrait du Working Paper, $n^{\circ} 189$ :

"White Privilege and Male Privilege : A Personal Account of Coming To See Correspondences through Work in Women's Studies", Wellesley College Center for Research on Women, 1988.

22. Fraser, Nancy, Qu'est-ce que la justice sociale ? Reconnaissance et redistribution, coll. Textes à l'appui, La Découverte, Paris, 2005. 